\title{
Photoemission of Pairs of Electrons from Rare-Gas Solids
}

\author{
H. W. Biester, ${ }^{(1)}$ M. J. Besnard, ${ }^{(2)}$ G. Dujardin, ${ }^{(2)}$ L. Hellner, ${ }^{(2)}$ and E. E. Koch ${ }^{(1)}$ \\ ${ }^{(1)}$ Fritz-Haber-Institut der Max-Planck-Gesellschaft, D-1000 Berlin 33, Federal Republic of Germany \\ ${ }^{(2)}$ Laboratoire de Photophysique Moléculaire, Université de Paris-Sud, 91405 Orsay, France \\ (Received 5 May 1987)
}

\begin{abstract}
By using a new photoelectron-photoelectron coincidence technique, we demonstrate that the absorption of vacuum-ultraviolet photons $(20<\hbar \omega<60 \mathrm{eV})$ by rare-gas solids leads to the ejection of pairs of electrons in addition to the well-known single-electron photoemission process. We show that at least two separate processes produce pairs of electrons with a surprisingly high efficiency.
\end{abstract}

PACS numbers: $32.80 . \mathrm{Fb}, 32.90 .+\mathrm{a}, 34.80 . \mathrm{Dp}, 79.60 .-\mathrm{i}$

One photon can interact with only one electron at once, so that photoemission of pairs of electrons, i.e., the absorption of a single photon followed by the ejection of two valence electrons, is a forbidden process unless the electron-electron Coulomb interaction is taken into account. Photoemission of pairs of electrons from the valence bands has not yet been observed in solids although it is known to occur in isolated atoms, ${ }^{1}$ molecules, ${ }^{2,3}$ and clusters. ${ }^{4}$ Such processes have to be distinguished from the Auger process ${ }^{5,6}$ in which a core and an Auger electron are emitted with well separated and discrete kinetic energies. Photoemission of pairs of electrons is a priori more difficult to study, both experimentally and theoretically, than the Auger processes because in this case the ejected electrons are indistinguishable and have a nondiscrete kinetic-energy spectrum.

We report in this Letter the first observation of photoemission of pairs of electrons from solids. Rare-gas solids ( $\mathrm{Ar}, \mathrm{Kr}$, and $\mathrm{Xe}$ ) were chosen because the van der Waals interatomic interaction is only weak. This makes their ionization energies comparable to the corresponding isolated atoms. ${ }^{7}$ Furthermore, we know that double photoionization is very efficient in the gas phase. ${ }^{8}$ One can thus expect that photoemission of pairs of electrons will also occur in rare-gas solids.

Synchrotron radiation from the DORIS II electron storage ring at DESY (Deutsches Elektronen-Synchrotron, Hamburg) dispersed by a 3-m normal-incidence monochromator was used as a photon source of variable energy in the 20-56-eV-energy range. An ultrahighvacuum chamber was equipped with a liquid-helium flow cryostat. ${ }^{9}$ Rare gases were condensed at low temperature $(\simeq 10 \mathrm{~K})$ on a copper substrate with film thickness of about $300 \mathrm{~nm}$. A new double time-of-flight electron spectrometer (see Fig. 1), which is described in detail elsewhere, ${ }^{10}$ was used to measure the number of photoelectron-photoelectron coincidences. Each of the two time-of-flight spectrometers was operated in a nonselective mode with a wide entrance aperture so that electrons could be detected with a high efficiency. The signal from one of the collectors was fed to the start input of a timeto-amplitude converter and the signal from the other collector was sent to the stop input after being delayed by about 350 ns. The number of false coincidences arising from the detection of two uncorrelated electrons produced by the absorption of two photons during the same radiation pulse of the storage ring is considered to be equal to 2 times the number of coincidences due to the detection of two electrons produced during two separate synchrotron radiation pulses. The number of true photoelectron-photoelectron coincidences $N_{c}^{t}$ was then obtained by the subtraction of 2 times the area of peak 2 from that of peak 1 in the photoelectron-photoelectron coincidence curve (see Fig. 2).

The results are shown in Fig. 3. Here the ratio $N_{c}^{t} / N_{e}$ ( $N_{c}^{t}$ is the number of coincidences and $N_{e}$ is the total number of electrons detected by each detector) is plotted as a function of the photon energy for solid xenon, krypton, and argon. We see a steep increase in the $\mathrm{Xe}, \mathrm{Kr}$, and Ar curves at 20.9, 25, and $28.6 \mathrm{eV}$, respectively, followed by a decreasing part and a second smooth increase starting at 30,36 , and $40 \mathrm{eV}$, respectively. The origin of these two sets of threshold energies can be understood after examination of the data in Table I.

The lower set of threshold energies clearly corresponds to the energies which are necessary to extract two $p$ electrons from two separate rare-gas atoms. This process,

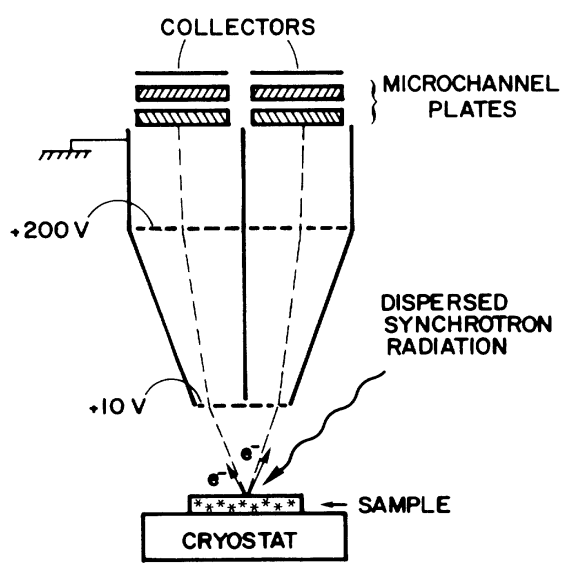

FIG. 1. Schematic diagram of the photoelectron-photoelectron coincidence experiment. 


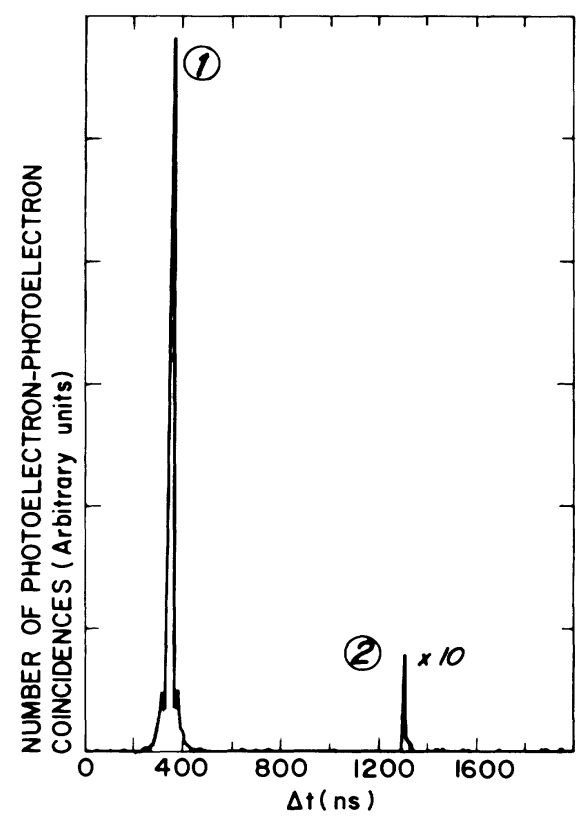

FIG. 2. Number of coincidences as a function of the difference $\Delta t$ between the time of flight of the two photoelectrons. This curve was recorded from a Xe film with photons of $27 \mathrm{eV}$. Peak 1 corresponds to true plus false coincidences. Peak 2 corresponds to false coincidences only (see text) and is multiplied by 10 in the figure in order to be observable. The separation between two synchrotron radiation pulses was $960 \mathrm{~ns}$.

which will be called secondary photoemission of pairs of electrons or secondary double photoemission, consists of the absorption of a photon by one atom followed by the ejection of an electron which has sufficient kinetic energy to ionize a second atom by electron-electron scattering [see Fig. 4(a)]. Both the primary and the secondary electrons are ejected from the solid and detected in coincidence. One can assume in first approximation that the onset energy of the secondary double photoemission is the largest of the two quantities 2[I.P. $(p)$ ] and $2 E_{\text {gap }}(p)$ depending on whether the electron affinity is positive or negative. The predicted threshold energy of this process is thus $19.6,23.8$, and $28.4 \mathrm{eV}$ for $\mathrm{Xe}, \mathrm{Kr}$, and $\mathrm{Ar}$, respectively, which is in relatively good agreement with the experimental values of $20.9,25.0$, and $28.6 \mathrm{eV}$. At the present state it is difficult to decide whether the slight difference between these two sets of values is due to the way in which the threshold energies have been measured or to a small significant deviation from the abovementioned predicted rule. This first onset of electron pair photoemission also coincides by chance, with the energies for single ionization from the outer $s$ band (see Table I) which could induce additional interatomic processes. However, the excitation of the $s$ electrons is believed to be negligible since its oscillator strength is very small. ${ }^{13}$ We emphasize that the observed secondary double photoemission is a very intense process since the

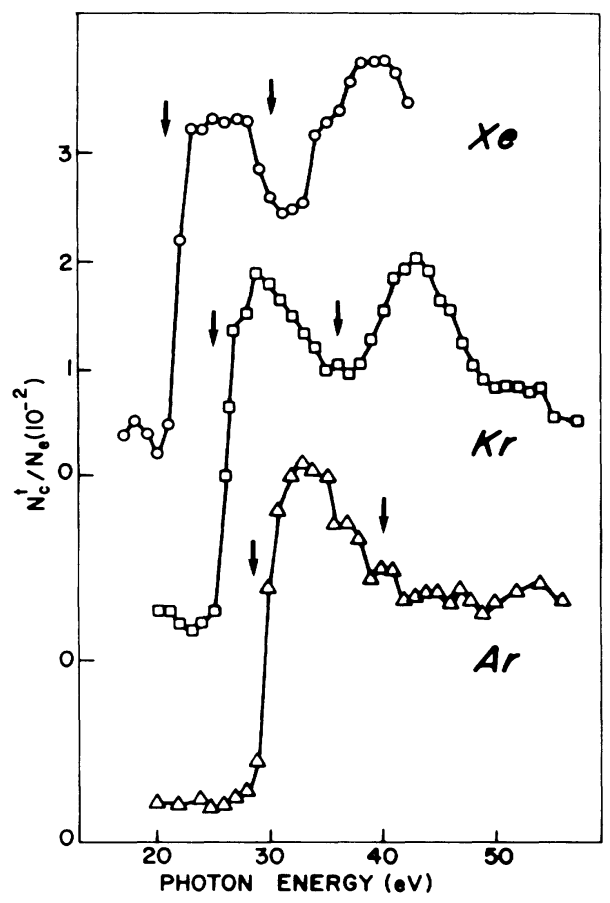

FIG. 3. Ratio of the number of true coincidences $N_{c}^{t}$ to the number of electrons detected by each detector $N_{e}$ as a function of the photon energy for films of xenon, krypton, and argon with a thickness of $300 \mathrm{~nm}$. The arrows indicate the experimental onset energies of the double photoemission processes. The second onset was determined by the change of the slope of the experimental curves.

TABLE I. Energies (in electronvolts) of electronic processes in rare-gas solids: $E_{\text {gap }}(p), p$-band gap energy; $E_{\text {aff }}$, electron affinity; I.P. $(p)$ and I.P. $(s)$, ionization energy from the $p$ and $s$ bands, respectively. In this table $\operatorname{Max}\{A, B\}$ refers to the largest of the two quantities $A$ and $B$.

\begin{tabular}{|c|c|c|c|}
\hline & $\mathrm{Xe}$ & $\mathrm{Kr}$ & $\mathrm{Ar}$ \\
\hline$E_{\text {gap }}(p)^{\text {a }}$ & 9.3 & 11.6 & 14.2 \\
\hline$E_{\text {aff }}^{\text {a }}$ & 0.5 & 0.3 & -0.4 \\
\hline I.P. $(p)^{\mathrm{a}}$ & 9.8 & 11.9 & 13.8 \\
\hline $\operatorname{Max}\left\{2[\operatorname{I} . P .(p)], 2 E_{\mathrm{gap}}(p)\right\}$ & 19.6 & 23.8 & 28.4 \\
\hline $\begin{array}{l}\text { First onset of electron pair } \\
\text { photoemission }^{b}\end{array}$ & 20.9 & 25.0 & 28.6 \\
\hline $\operatorname{I.P}(s)^{\mathrm{c}}$ & 20.5 & 24.5 & 28.4 \\
\hline $\begin{array}{c}\operatorname{Max}\{\operatorname{IIP} .(p)+\operatorname{IIP} .(s) \\
\left.E_{\text {gap }}(p)+E_{\text {gap }}(s)\right\}\end{array}$ & 30.3 & 36.4 & 43.0 \\
\hline $\operatorname{Max}\left\{3[\right.$ I.P. $\left.(p)], 3 E_{\mathrm{gap}}(p)\right\}$ & 29.4 & 35.7 & 42.6 \\
\hline $\begin{array}{l}\operatorname{Max}\left\{2.8 E_{\mathrm{gap}}(p)+2 E_{\mathrm{aff}}\right. \\
\left.2.8 E_{\mathrm{gap}}(p)\right\}\end{array}$ & 27.0 & 33.1 & 39.8 \\
\hline $\begin{array}{l}\text { Second onset of electron pair } \\
\text { photoemission b }\end{array}$ & $\leq 30$ & $\leq 36$ & $\leq 40$ \\
\hline
\end{tabular}

${ }^{a}$ Reference 11.

${ }^{\mathrm{b}}$ This work.

'Reference 12. 
(a)

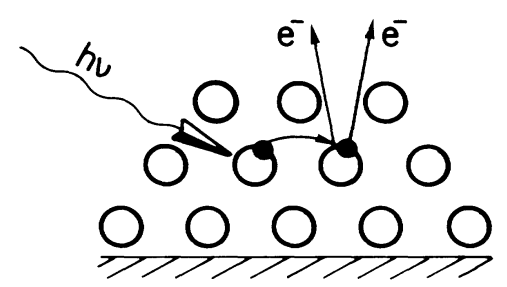

(b)

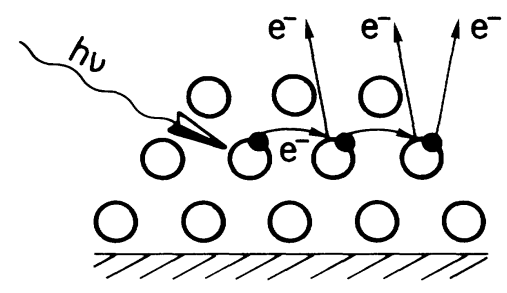

(c)

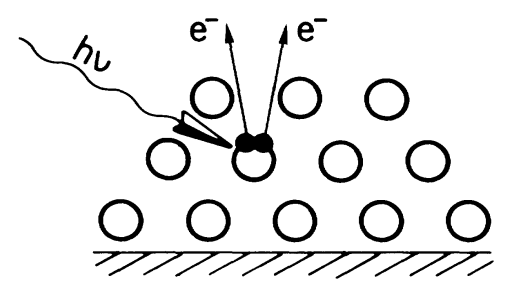

FIG. 4. Schematic representation of the multiple photoionization processes in rare-gas solids: (a) secondary double photoemission, (b) three-atom process, and (c) direct double photoemission.

first maxima of the curves in Fig. 3 correspond to $N_{c}^{t} / N_{e}$ values as high as $3.3 \times 10^{-2}$. Considering that the probability for an ejected electron to reach a given detector is less than or equal to 0.08 (Ref. 10) and that the detection efficiency of each detector is about 0.5 , this maximum value of $N_{c}^{t} / N_{e}$ corresponds to a ratio of the cross section for ejection of pairs of electrons to the total photoemission cross section of about $80 \%$, which represents a very high proportion of pairs of electrons.

From the results of Schwentner ${ }^{13}$ it is known that the mean free path of the primary electrons produced at the onset photon energy of the double photoemission is around $10 \AA$ for $\mathrm{Xe}, \mathrm{Kr}$, and Ar. These distances are approximately equal to the smallest distances between atoms in the corresponding solids, ${ }^{14}$ which indicates that the pairs of electrons originate from neighboring atoms or atoms close by. We note in passing that electronelectron scattering processes have been the subject of numerous experimental and theoretical studies in semiconductors, ${ }^{15}$ metals, ${ }^{16}$ and organic crystals. ${ }^{17}$ More recently similar secondary effects of photoelectrons, for producing pairs of photoelectrons and photons detected in coincidences in anthracene crystals, have also been observed by Klein. ${ }^{18}$

The second process for ejection of pairs of electrons with onset energies of about 30,36, and $40 \mathrm{eV}$ for $\mathrm{Xe}$,
$\mathrm{Kr}$, and $\mathrm{Ar}$, respectively, may be ascribed, on the basis of the data in Table I, to one or several of the three following effects:

(i) The ejection of two electrons from the outermost $s$ and $p$ levels of two separate atoms. We know from gasphase experiments ${ }^{19}$ that, for example, in xenon the ratio $\sigma(5 s) / \sigma(5 p)$ of the partial ionization cross sections is extremely small all over the $25-40-\mathrm{eV}$ photon-energy range. Extrapolating these results to solids allows us to consider that the ejection of an $s$ electron with enough kinetic energy to ionize another atom is a very unlikely process in this photon-energy range. On the other hand, we cannot completely exclude that a $p$ electron would be first ejected in order to ionize a second atom from the $s$ level.

(ii) The ejection of three electrons from three separate atoms, i.e., ejection, after photon absorption, of a primary electron followed by ejection of a second electron by electron-electron scattering and ejection of a third electron by electron-electron scattering on a third atom [see Fig. 4(b)]. In such three-collision processes we would not expect to see a large difference between Xe, $\mathrm{Kr}$, and $\mathrm{Ar}$ since the secondary double photoemission which is a two-collision process does not show any significant change of intensity when going from $\mathrm{Xe}$ to Ar.

(iii) The third possible process consists of the ejection of two electrons from the same atom due to the intraatomic electron-electron interaction [see Fig. 4(c)].

It is known from the gas phase that these processes have threshold energies at about 2.8 times the threshold energy of the single photoionization process. ${ }^{2,3,8}$ The application of the same empirical rule to the solid rare gases leads to threshold energies of 27.0, 33.1, and 39.8 $\mathrm{eV}$ for $\mathrm{Xe}, \mathrm{Kr}$, and $\mathrm{Ar}$, respectively. These values are quite compatible with the experimental values for the onset energies of the second process for formation of pairs of electrons. This direct (or primary) double photoemission process, when observed in the gas phase,${ }^{8}$ has a relative efficiency as compared to the total photoemission which decreases when going from $\mathrm{Xe}$ to $\mathrm{Kr}$ and Ar. A similar trend is observed by us experimentally for the solid phase if we consider the height of the second maxima of the curves in Fig. 3. It follows that the second process for formation of pairs of electrons most probably corresponds to a direct double photoemission of the same type as that observed in isolated atoms, ${ }^{8}$ although we cannot exclude that other processes, such as the threeelectron photoemission or the double photoemission involving the $s$ level, would also occur at higher photon energies. Further experiments using the kinetic-energyselective mode of the electron time-of-flight spectrometers will help us to distinguish between these processes.

In conclusion, the existence of photoemission of pairs of electrons in solids has been clearly demonstrated in rare-gas solids. At least two separate processes have been shown to exist with a surprisingly high efficiency. 
This electron-pair emission seems to be a specific probe of electron-electron correlations and electron-electron scattering processes in solids.

Two of us (G.D. and L.H.) would like to thank the staff at Hamburger Synchrotronstrahlungslabor for the great hospitality during the measurements and the Max-Planck-Gesellschaft for financial support. We are indebted to $\mathrm{V}$. Saile for his encouragement and very helpful discussions and to $\mathrm{S}$. Leach for his continuing interest and critical reading of the manuscript.

${ }^{1}$ T. A. Carlson, Phys. Rev. 156, 142 (1967).

${ }^{2}$ B. P. Tsai and J. H. D. Eland, Int. J. Mass Spectrom. Ion Phys. 36, 143 (1980).

${ }^{3}$ G. Dujardin, S. Leach, O. Dutuit, P. M. Guyon, and M. Richard-Viard, Chem. Phys. 88, 339 (1984).

${ }^{4}$ C. Brechignac, M. Broyer, Ph. Chauzac, G. Delacretaz, P. Labastie, and L. Wöste, to be published.

${ }^{5}$ H. W. Haak, G. A. Sawatzky, and T. D. Thomas, Phys. Rev. Lett. 41, 1825 (1978).

${ }^{6}$ H. W. Haak, G. A. Sawatzky, L. Ungier, J. K. Gimzewski, and T. D. Thomas, Rev. Sci. Instrum. 55, 696 (1984).
${ }^{7}$ N. Schwentner, E. E. Koch, and J. Jortner, in Electronic Excitations in Condensed Rare Gases, edited by G. Höhler, Springer Tracts in Modern Physics, Vol. 107 (Springer-Verlag, Berlin, 1985).

${ }^{8}$ J. A. R. Samson and G. N. Haddard, Phys. Rev. Lett. 33, 875 (1974).

${ }^{9}$ N. Schwentner, A. Harmsen, E. E. Koch, V. Saile, and M. Skibowski, in Vacuum Ultraviolet Radiation Physics, edited by E. E. Koch, R. Haensel, and C. Kunz (Pergamon, New York, 1974).

${ }^{10}$ H. W. Biester, G. Dujardin, L. Hellner, and E. E. Koch, to be published.

${ }^{11}$ N. Schwentner, F. J. Himpsel, V. Saile, M. Skibowski, W. Steinman, and E. E. Koch, Phys. Rev. Lett. 34, 528 (1975).

${ }^{12} \mathrm{~S}$. Bernstorff, thesis, DESY Internal Report No. Desy F41, Hasylab 84-03, 1984 (unpublished).

${ }^{13}$ N. Schwentner, Phys. Rev. B 14, 5490 (1976).

${ }^{14}$ G. L. Pollack, Rev. Mod. Phys. 36, 748 (1964).

${ }^{15}$ E. O. Kane, Phys. Rev. 159, 624 (1967).

${ }^{16} \mathrm{C}$. N. Berglund and W. E. Spicer, Phys. Rev. 136, 1030 (1964).

${ }^{17}$ K. Seki, T. Hirooka, Y. Kamura, and H. Inokuchi, Bull. Chem. Soc. Jpn. 49, 904 (1976).

${ }^{18}$ G. Klein, Chem. Phys. Lett. 95, 305 (1983).

${ }^{19}$ M. Y. Adam, thesis, Université Paris-Sud, Orsay, 1978 (unpublished). 